\title{
Rejoinder on: Multicriteria decision systems for financial problems
}

\author{
Constantin Zopounidis • Michael Doumpos
}

Published online: 4 May 2013

(C) Sociedad de Estadística e Investigación Operativa 2013

We are truly grateful to the colleagues who prepared their insightful comments on our paper. We should also express our gratitude to the editor-in-chief of TOP, Professor Jesús Artalejo, who invited us to contribute to the journal and handled the collection of the discussions in a very professional and timely manner.

All discussants have extensive research expertise with major contributions in both the theory of MCDA and its applications in finance. We feel confident that the readers of TOP will appreciate theses discussions, which highlight particular aspects of the contributions of multicriteria decision systems in finance, add new perspectives on the past and recent advances in this area, and raise important issues for future research. In this rejoinder, we shall briefly elaborate on these discussions and the alternative points of view introduced by the colleagues.

The first point that is immediately evident is that portfolio management (PM) serves as the main basis for most of the discussants' comments. This should not be a surprise, as PM is a multifaceted problem whose implications extend to many other financial domains, such as asset-liability management, financial planning, investment analysis, and credit portfolio management. As we elaborated in our review, and it

This rejoinder refers to the comments available at doi:10.1007/s11750-013-0276-X, doi:10.1007/s11750-013-0277-9, doi:10.1007/s11750-013-0278-8, doi:10.1007/s11750-013-0280-1, doi:10.1007/s11750-013-0281-0.

C. Zopounidis $(\varangle) \cdot$ M. Doumpos

Dept. of Production Engineering and Management, Financial Engineering Laboratory, Technical University of Crete, University Campus, 73100 Chania, Greece

e-mail: kostas@dpem.tuc.gr

M. Doumpos

e-mail: mdoumpos@dpem.tuc.gr

C. Zopounidis

School of Management, Audencia Group, Nantes, France 
is further indicated by the discussants' comments, PM involves among others asset screening, evaluation, and selection, the analysis of risk aversion and the management of uncertainty, the statistical analysis of risk-return patterns, the optimization of asset allocations, as well as the management of the constructed portfolios (e.g., rebalancing and trading strategies).

Issues relevant to asset screening and selection are discussed by Greco, Matarazzo, and Słowiński, as well as Pla-Santamaria and Garcia-Bernabeu. Pla-Santamaria and Garcia-Bernabeu emphasize that not all financial decision makers adopt the same risk aversion attitude. As we explained in our review, this is indeed true as risk preferences cannot be universally defined. Nevertheless, the financial theory is often based on specific assumptions on the risk aversion attitude of financial decision makers, usually modeled (explicitly or implicitly) through predefined utility functions. Such a setting, however, provides limited flexibility and guidance in situations where particular risk preferences should be considered in the analysis. Furthermore, financial decision makers are not only characterized by different levels and conception of risk aversion, but their attitude toward risk changes dynamically over time in accordance with the sentiment in the markets and the state of the business environment. Multicriteria systems contribute in this context through the introduction of systematic approaches for modeling subjective and time-dependent risk preferences, which may expressed in various forms.

Aouni further emphasizes that the portfolio selection criteria considered by financial decision makers are conflicting. This is a fundamental point even in the traditional risk-return framework, which is based on risk and return, each of which naturally leads to different/conflicting choices (i.e., the maximization of return requires an investor to take higher risks and vise versa). The conflicts become much more involved when additional decision criteria are introduced in the analysis. As a consequence, financial decision makers search for the best acceptable compromise solutions based on their judgment policy and experience. Without the support of proper MCDA tools, this can only be done in an ad hoc empirical basis, which is likely to fail to identify interesting solutions (as noted in the comments of Steuer for a priori methods) and makes it impossible to formalize solid arguments in support of the results.

In our review, we indicated how different multicriteria systems and techniques contribute in resolving these limitations. In their comment, Greco, Matarazzo, and Słowiński focus on some recently developed MCDA techniques. First, they outline the contribution of symbolic models developed on the basis of the dominance-based rough set approach, which has been introduced by the discussants. This is indeed a very interesting approach as the resulting decision rule models are expressed in natural language and can be easily understood by investors, portfolio managers, and financial decision makers in general. Greco, Matarazzo, and Słowiński illustrate how this framework can be used as a tool to facilitate the modeling of the preferences of the investors toward risk and other portfolio selection criteria. In recent works, the authors have used this approach to describe uncertainty and risk with respect to the returns using the quantiles of the returns distribution. Such an approach is a positive contribution to the rich literature on quantile risk measures (Miller and Ruszczyński 2008) and it is worth further consideration in the context of the ongoing debate on coherent approaches for measuring financial risks (Artzner et al. 1999). 
Greco, Matarazzo, and Słowiński also discuss the usefulness of ordinal regression techniques. Such techniques are implemented in the context of preference disaggregation analysis (Jacquet-Lagrèze and Siskos 2001) and can be particularly useful tools for financial decision problems, including PM (Zopounidis et al. 1999). Such a regression-based framework for model building and preference modeling, reduces the cognitive effort required by financial decision makers with regard to the specification of preferential information (a problem that as Pla-Santamaria and GarciaBernabeu note is evident for other MCDA methods such as AHP). As we indicated in our review, preference disaggregation methods have already been successfully used in many areas of financial decision making. Nevertheless, it would be particularly interesting to analyze the effectiveness and the results obtained from the latest advances in this field (as outlined by Greco, Matarazzo, and Słowiński) for financial decision aiding, in areas such as PM, credit scoring, banking, country risk analysis, etc. Among the recent advances in this area, robust decision support is of particular interest. From a statistical perspective, robustness is mostly involved with the stability of statistical estimators and estimates, whereas from a financial perspective robustness is often assumed to refer to the distribution of the returns (Fabozzi et al. 2007). MCDA brings in a new perspective, focusing on the robustness and variations of the obtained recommendations due to the preferential information provided by the decision maker and his/her judgment policy. This brings a decision-theoretic point of view to robustness analysis, which is of particular interest for financial problems, where the preferences of the decision makers change over time, as explained above.

Of course, it should be noted that the tools available in the context of multicriteria systems, as well as related issues raised by the discussants cover a wide range of financial evaluation problems and are not only relevant to PM. For instance, in our review, we focused on corporate performance evaluation. Pla-Santamaria and Garcia-Bernabeu further refer to fund performance measurement, which in closely related to PM. In this context, Pla-Santamaria and Garcia-Bernabeu note the importance of distinguishing between the preferences of managers and investors in connection to the use of traditional fund appraisal criteria. It is true that in this framework, the finance theory has (mostly) focused on developing measures for evaluating the performance and abilities of fund managers. Such performance measures are well-established and they informative for professional fund managers. Facilitating an investor's decision, however, requires the consideration of additional criteria. For instance Babalos et al. (2012) considered expenses and front-end loads in combination with risk-return criteria and traditional measures of fund performance, whereas Tamiz et al. (2013) further used macroeconomic and country-specific criteria. On the other hand, Ballestero et al. (2012) and Pérez-Gladish and M'Zali (2010) introduced criteria related to social responsibility, which is an issue that has gained much interest recently among researchers and professionals. Thus, it is evident that the performance of funds cannot be solely described by the asset selection and market timing abilities of their managers, as an investor would also take into consideration other micro and macro factors that are relevant to his/her investment policy and the conditions of the investment environment. Of course, except for the fund evaluation measures, portfolios of funds can also be considered in a setting similar to PM as illustrated in several studies (see, for instance, Davies et al. 2009; Tamiz et al. 2013). 
On the algorithmic side, Steuer discusses the suitability of a posteriori vs. a priori methods. As he correctly notes, a posteriori approaches provide much richer information to financial decision makers, allowing them to explore different good options, based on the identification of the full efficient frontier. With the significant improvements achieved on the algorithmic side and the computational power now available, we think that this is indeed a feasible approach. The introduction of powerful metaheuristics for multiobjective optimization has been really helpful in this context, particularly for complex financial optimization problems (e.g., portfolio optimization with cardinality constraints, the introduction of complex risk measures and portfolio selection criteria). In fact, such methods have gained much popularity with numerous applications in PM and other areas in finance (Maringer 2005). On the other hand, interactive techniques have also evolved over the years and promising new techniques have emerged, such as the ones briefly outlined by Greco, Matarazzo, and Słowiński (i.e., the combination of the dominance-based rough set approach with multiobjective techniques). Again, it should be noted that the computational and algorithmic advances enable the application of both interactive and a posteriori techniques even for big financial data sets, as correctly noted by Aouni. The same also applies to preference disaggregation and ordinal regression techniques, which can also be employed to build financial decision models from big data, even for complex outranking models (Doumpos and Zopounidis 2011).

Nevertheless, aside from the actual relevance of multicriteria decision systems in financial problems, and the methodological and algorithmic advances, there is the issue of their use in practice. This is indeed a crucial point as discussed by PlaSantamaria and Garcia-Bernabeu, who note two main issues. The first involves the quantitative background of professionals in finance, whereas the second involves the relationship of multicriteria approaches to financial theory. As far as the first issue is concerned, it should be acknowledged that over the years the quantitative background of professional financial analysts and decision makers has strengthened, following the increasing complexity and sophistication of financial models. Nowadays, in many cases (e.g., financial engineers and risk analysts), a strong background on areas such as statistics, econometrics, optimization, stochastic processes, simulation, and computer science, is considered as an important qualification. In that regard, multicriteria systems can also be considered as feasible choices. In our view what is really important, is that the applications and implementations of multicriteria approaches are grounded not only on solid decision-theoretic bases, but also be financially relevant. As we elaborated in our review, the financial theory provides a rich set of normative and descriptive models as well as theoretical and empirical results. MCDA approaches should build on these grounds, acting as complementary and enhancing tools, focusing on the new perspectives that they bring (e.g., constructive approach) and emphasizing their decision-aiding orientation. This is not enough, however, as the realism and the appealing features of multicriteria systems for financial problems, should be combined with positive comparative results and rigorous model validation tests. This is of outmost importance, given that the financial regulatory framework becomes stricter as far as it concerns the validity and effectiveness of the models used to support financial decisions. This issue is noted by Andriosopoulos in the case of credit scoring, but it is actually relevant in all areas of financial risk management. 
Even though not all types of financial decisions are under supervisory control (as in the case of financial institutions), this is still an important decisive factor that cannot be overlooked. Thus, closing the gap between the decision-aid orientation of MCDA and the principles of financial theory, together with the scaling of MCDA techniques to large data and the availability of financial multicriteria decision support systems and free computer codes are decisive factors for the strengthening of the adoption of multicriteria systems in financial practice.

\section{References}

Artzner P, Delbaen F, Eber JM, Heath D (1999) Coherent measures of risk. Math Finance 9(3):203-228

Babalos V, Philippas N, Doumpos M, Zopounidis C (2012) Mutual funds performance appraisal using stochastic multicriteria acceptability analysis. Appl Math Comput 218(9):5693-5703

Ballestero E, Bravo M, Pérez-Gladish B, Arenas-Parra M, Pla-Santamaria D (2012) Socially responsible investment: a multicriteria approach to portfolio selection combining ethical and financial objectives. Eur J Oper Res 216(2):487-494

Davies RJ, Kat HM, Lu S (2009) Fund of hedge funds portfolio selection: a multiple-objective approach. J Deriv Hedge Funds 15(2):91-115

Doumpos M, Zopounidis C (2011) A multicriteria outranking modeling approach for credit rating. Decis Sci 42(3):721-742

Fabozzi FJ, Kolm PN, Pachamanova D, Focardi SM (2007) Robust portfolio optimization and management. Wiley, New York

Jacquet-Lagrèze E, Siskos Y (2001) Preference disaggregation: twenty years of MCDA experience. Eur J Oper Res 130(2):233-245

Maringer D (2005) Portfolio management with heuristic optimization. Springer, Dordrecht

Miller N, Ruszczyński A (2008) Risk-adjusted probability measures in portfolio optimization with coherent measures of risk. Eur J Oper Res 191(1):193-206

Pérez-Gladish B, M'Zali B (2010) An AHP-based approach to mutual funds' social performance measurement. Int J Multicriteria Decis Mak 1(1):103-127

Tamiz M, Azmi RA, Jones DF (2013) On selecting portfolio of international mutual funds using goal programming with extended factors. Eur J Oper Res 226(3):560-576

Zopounidis C, Doumpos M, Zanakis S (1999) Stock evaluation using a preference disaggregation methodology. Decis Sci 30(2):313-336 